Technological University Dublin ARROW@TU Dublin

2008

\title{
Novel Calixarene-Schiff Bases that Bind Silver(I) Ion
}

\author{
Mary Deasy \\ Technological University Dublin, mary.deasy@tudublin.ie \\ Bernadette Creaven \\ Technological University Dublin, bernadette.creaven@tudublin.ie \\ Brian A. Murray \\ Technological University Dublin, brian.murray@tudublin.ie
}

See next page for additional authors

Follow this and additional works at: https://arrow.tudublin.ie/ittsciart

Part of the Chemistry Commons

\section{Recommended Citation}

Deasy, M., Creaven, B. \& Murray, B. (2008). Novel Calixarene-Schiff Bases that Bind Silver(I) Ion. Inorganic Chemistry Communications vol. 11, no. 10, pg. 1215-1220. doi:10.1016/j.inoche.2008.06.011

This Article is brought to you for free and open access by the School of Science and Computing at ARROW@TU Dublin. It has been accepted for inclusion in Articles by an authorized administrator of ARROW@TU Dublin. For more information, please contact arrow.admin@tudublin.ie, aisling.coyne@tudublin.ie, gerard.connolly@tudublin.ie.

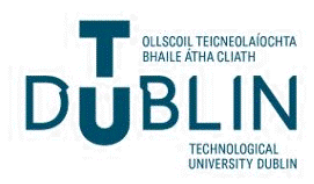




\section{Authors}

Mary Deasy, Bernadette Creaven, Brian A. Murray, John McGinley, and Paul M. Flood 


\title{
Novel Calixarene-Schiff Bases that Bind Silver(I) Ion
}

\author{
Bernadette S. Creaven ${ }^{\mathrm{a}}$, Mary Deasy ${ }^{\mathrm{a}}$, Paul M. Flood ${ }^{\mathrm{a}}$, John McGinley ${ }^{\mathrm{b}}$, and Brian A. Murray*,a \\ ${ }^{a}$ Department of Science and National Centre for Sensor Research, Institute of Technology Tallaght, \\ Tallaght, Dublin 24, Ireland; \\ ${ }^{b}$ Department of Chemistry, the National University of Ireland-Maynooth, Co. Kildare, Ireland.
}

\begin{abstract}
Two novel calix[4]arene-Schiff base receptors have been synthesized. One of the new compounds has two pendant aldimines, while the second has been prepared by two-point attachment of a calixarene-dialdehyde onto a calixarene-diamine to form a "calix-tube". Preliminary binding studies with $\mathrm{AgClO}_{4}$ show large complexation-induced shifts in ${ }^{1} \mathrm{H}$ NMR positions.
\end{abstract}

Keywords: calixarenes, Schiff base, silver, complexation 


\section{Introduction}

Calixarenes have proved popular building blocks for the development of highly specific synthetic receptors particularly for ionic guest species. ${ }^{1}$ They have found widespread use in sensor technology, as sensing agents for various analytes. ${ }^{2}$ Calix [n]arenes are macrocyclic compounds in which phenolic units are linked via methylene bridging groups at their ortho positions. The spectacular development of these well defined macromolecular systems in recent years is related to the ease with which the upper (aryl) and lower (phenolic) rims have been modified in a stereocontrolled and regiocontrolled manner, ${ }^{1}$ coupled with the wide range of cationic ${ }^{3,4}$ and neutral ${ }^{5-7}$ guests they have been found to bind.

The smallest in the series is where four phenolic units make up the macrocyclic backbone $(n=4)$. This offers a highly rigid platform on which to attach functional groups with potential to act as preorganised binding sites for selective complexation, hence its use by many research groups as a key structural motif for the design and synthesis of an ever increasing number of synthetic receptors. ${ }^{8}$ Higher order calix[4]arenes have been designed and synthesised, where either the lower or upper cavity has been bridged by crown, ${ }^{9}$ azacrown ${ }^{10}$ and peptide ${ }^{11}$ moieties to expand the range of guests bound. Several double calix[4]arenes (or calix-tubes) ${ }^{12}$ have been prepared as additional examples of more complex molecular architectures in the recent past.

The attachment of N-ligating groups through imine bond formation has been successful at the upper ${ }^{13}$ and lower ${ }^{14}$ rim of calixarenes. This has resulted in the formation of calixarene-Schiff base receptors which have shown potential for cation recognition, particularly with the transition metals. ${ }^{15}$ Herein we report the efficient syntheses of two new calixarene-Schiff base compounds along with some preliminary binding results. One of the new compounds is a double calixarene (or calix-tube) where the two calixarene units are linked at their lower rims (tail-to-tail) ${ }^{16}$ using imine spacer groups. Both new compounds are distal functionalised calix[4]arenes, i.e. where the 1,3-phenolic $\mathrm{H}$ atoms have been replaced. In addition a new calixarene-dialdehyde (calix-dial) template was isolated in the cone conformation. 
1,3-Substitution on two of the oxygens on the lower rim of calix[4]arene is readily achieved by alkylation, and McKervey has prepared and studied many such derivatives and their binding properties to cations ${ }^{17}$ : they typically retain the cone conformation, easily identified by ${ }^{1} \mathrm{H}-\mathrm{NMR}$ spectroscopy. ${ }^{18}$ Many more recent examples use such methodology: ${ }^{19}$ Bagatin has employed 1,3-dibromopropane to give a 1,3-bis(bromopropyl) derivative (1, Scheme 1$)$ of the parent tetra-t-butylcalix[4]arene,${ }^{20}$ an alkylation which leaves two pendant bromines for further elaboration. Subsequent reaction with two moles of 8-hydroxyquinoline was used to develop a $\mathrm{Zn}^{2+}$ receptor.

Calixarenes with pendant 1,3-distal benzaldehyde groups are known, ${ }^{21}$ and have recently found use as templates in the development of stilbene-bridged calixarene photoswitchable molecular receptors. ${ }^{22}$

Calixarenes bearing aldehyde moieties also provide an ideal platform for the synthesis of Schiff base calixarenes, which have attracted recent interest. Liu, for example, has attached two azosalicylaldehyde units to calix[4]arene by means of imine chemistry on a diamino-calixarene, ${ }^{23}$ and has then followed their binding to alkali metal cations by UV. Pendant Schiff bases, of course, also open new metal targets for calixarenes: Vicens has used a diimine-bridged calixarene to extract d- and f-block metal cations, ${ }^{24,25}$ Liu has complexed pendant vanillin-derived imines with seven lanthanide trications, ${ }^{26}$ and Mahajan has bound silver using calixarenes joined together by diimine links in the development of silver-selective electrodes. ${ }^{27,28}$

We have reacted dibromide (1) with 3-hydroxybenzaldehyde to give (2), a new distal 1,3calixarene derivative with pendant aldehydes at the lower rim (a calix-dial). ${ }^{29}$ Using 3 -aminopyridine as a representative amine that would also introduce an additional chelatable nitrogen, we attempted to react it with dial (2) to give calix-Schiff derivative (3), using standard aldimine-forming conditions (ethanolic reflux). This was, however, unsuccessful. Going back a step to the dibromide (1) and reacting it with a pre-formed Schiff base (4) - prepared from the amine and 3-hydroxybenzaldehyde ${ }^{30}$ - gave the desired calixarene with two pendant Schiff bases (3) in $66 \%$ yield (Scheme 1). ${ }^{31}$

Returning to dial (2), we could see the potential for it to react with a diamine, to give a bridged structure. Indeed, if the bridge itself was a calixarene, the product would be a calix-tube, a large-ring structure containing two calixarenes joined by two links. Examples of such receptors include 
McKervey's diallyl-tube as a Grubb's catalyst, ${ }^{32}$ Beer's ethano-linked $\mathrm{K}^{+}$-selective calixarenes, ${ }^{33}$ and Kim's crown-calix-tube with glycolic links. ${ }^{34}$

Double-condensation of calix-diamine (5) - prepared by Wolf's method ${ }^{35}$ - with calix-dial (2) gave calix-tube (6) in $89 \%$ yield (Scheme 2), under simple conditions $\left(\mathrm{MeOH} / \mathrm{Et}_{3} \mathrm{~N}\right.$ reflux overnight). ${ }^{36}$

The contrast in the reactivities of the aldehyde (2) with amines — viz. unreactive with 3aminopyridine (EtOH reflux) but giving clean imine product (6) with calix-diamine (5) (MeOH reflux) - is noteworthy. It presumably is due to the lower nucleophilicity of 3-aminopyridine (aromatic amine with electron-withdrawing group) compared to the primary alkylamine groups of (5).

Thus we have prepared two novel Schiff base calix[4]arenes via convenient syntheses which should be easily generalized. The first (compound 3), with two 1,3-pendant imine functions, was obtained from tetra-t-buylcalix[4]arene in two steps. The second, a calix[4]-calix[4]-tube with 1,1'and 3,3'-imine links (compound 6), also required two steps from the same reactant to produce its immediate precursors, and one further step to join them.

Preliminary complexation studies were carried out on the two new calixarene compounds, calixSchiff (3) and calix-tube (6). The complexes were prepared by recrystallization over a few days from acetonitrile solutions that were equimolar in $\mathrm{AgClO}_{4}$ and receptor. Although crystals suitable for Xray crystallography were not isolated, the solids obtained showed evidence of complexation in $\mathrm{CDCl}_{3}$ solution (see binding studies, next paragraph). That the solids, too, are complexes (rather than precipitated reactants) is suggested by their complete and immediate dissolution in chloroform, as free $\mathrm{AgClO}_{4}$ is not soluble in this solvent at the concentrations employed.

Initial binding studies were carried out in $\mathrm{CDCl}_{3}$ by $\mathrm{NMR}$, with silver(I) complexes having been chosen as the cation is known to bind imines but wouldn't interfere with NMR, and the structure of the ligands provides many opportunities for complexation-induced chemical shift changes.

${ }^{1} \mathrm{H}$ NMR (Figure 1) indicated that many peaks were significantly shifted by silver(I) complexation, particularly the imine ( $\underline{\mathrm{HC}}=\mathrm{N}: 8.27$ to $8.37 \mathrm{ppm})$ and $\mathrm{OH}$ signals (7.76 to $7.94 \mathrm{ppm})$, 
with downfield movements also seen for both of the calixarene aromatic signals, the $t$-butyls, the calixarene methylene doublets $(+0.07$ and $+0.10 \mathrm{ppm})$, and the aromatic signals derived from both the amine and the aldehyde, the latter also becoming noticeably broadened. There were some upfield shifts too: the methylene nearest the Schiff base $\left(\underline{\mathrm{C}}_{2} \mathrm{OPhCH}=\mathrm{N}\right)$ moves $-0.19 \mathrm{ppm}$. These changes indicate not merely that $\mathrm{Ag}^{+}$has been bound, but that it has brought about a profound structural change affecting the whole molecule.

Calix-tube (6) shows similar - indeed larger $-{ }^{1} \mathrm{H}-\mathrm{NMR}$ complexation-induced shifts with silver (Figure 2). The most notable shifts were those of the imine function $(+0.40 \mathrm{ppm})$, and the OH's of both calixarene units $(+0.18$ and +0.59 for the 'aldehyde' and 'amine' ends, respectively). For this molecule, the separation of the more downfield $t$-butyl signals at 1.24 and $1.25 \mathrm{ppm}$ increases by 0.06 ppm: these signals have been assigned to the $t$-butyl groups where the $O$-substitution took place. These more dramatic changes probably reflect the greater reorganization that has to accompany complexation of the silver cation in the more constrained calix-tube architecture: the cavity, made up of two calixarenes and two Schiff bases, may have to expand to allow the cation to enter, so forcing an inward movement of the $t$-butyls.

Comparing and contrasting the silver(I) interaction with the two receptors, it is notable that although calix-Schiff (3) does have 2 free hydroxyls on the calixarene, this are not optimally placed for binding if silver is coordinated by imine. The complexation-induced shift (CIS) is only $+0.18 \mathrm{ppm}$, which may be due to structural rearrangement, rather than direct binding. But calix-tube (6) may bind the cation by both imine nitrogen and hydroxyl, using the 'amine' hydroxyl (lower calix of 6). This hydroxyl moves more dramatically (CIS $=+0.59$ ), while the 'upper' $\mathrm{OH}$ only moves +0.18 , as it does in complexation of (3). This is also consistent with the other more dramatic changes seen for (6).

We intend to extend the binding studies to other metal cations, and in the case of calix-tube (6), the binding of a second (different) metal ion may be possible. More generally, there is considerable scope both to extend the range of imine structures accessible, and to further derivatize them. 


\section{Acknowledgements}

BAM \& BSC acknowledge the Irish Higher Education Authority for its funding of the NCSR,

including JMcG's post-doctoral fellowship. PMF thanks Enterprise Ireland, the Postgraduate R\&D Skills Programme, and ITT-Dublin's PhD Continuation Fund for grant support. 


\section{References}

1. (a) Gutsche C. D., Calixarenes Revisited; Stoddart J. F., ed., The Royal Society of Chemistry, Cambridge, UK, 1998 and references cited therein; (b) Review covering 2000-2007: Creaven B.S., Donlon D. F., McGinley J.; "Coordination Chemistry of calix[4]arene derivatives with lower rim functionalization and their Applications", Coord.Chem. Rev. (accepted 10 June 2008: MS = CCR-D-08-00029R1).

2. (a) Tuntulani T., Thavornyutikarn P., Poompradub S., Jaiboon N., Ruangpornvisuti V., Chaichit N., Asfari Z., Vicens V., Tetrahedron, 2002, 58, 10277; (b) Diamond D., McKervey M.A., Chem. Soc. Rev., 1996, 25, 15; (c) Kim S., Lee S.H., Lee J.Y., Bartsch R.A., Kim J.S., J. Am. Chem. Soc., 2004, 126, 16499; (d) Baklouti L., (asst. Ed.), Vicens J., Harrowfield J. (Eds.); “Calixarenes in the Nanoworld”, Springer, Dordrecht, 2007; (e) Tu, C., Surowiec, K., Bartsch, R. A.;

Tetrahedron 2007, 63, 4184; (f) Leyton P., Dimingo, C., Sanchez-Cortes S., Campos-Vallette M., Diaz G. F., Garcia-Ramos J. V.; Vib. Spectrosc. 2007, 43, 358; (g) Seyhan S., Colak M., Merdivan M., Demirel N., Anal. Chim. Acta, 2007, 584, 462.

3. Izatt R.M., Lamb J.D., Hawkins R T., Brown P.R., Izatt S.R., Christensen J. J., J. Am. Chem. Soc., 1983, 105, 1782.

4. Izatt S.R., Hawkins R.T., Christenesen J.J., Izatt R.M., J. Am. Chem. Soc., 1985, 107, 63.

5. Gutsche C.D., Dhawan B., No K.H., Muthukrishnan R., J. Am. Chem. Soc., 1981, 103, 3782.

6. Anreeti G.D., Ungaro R., Pochini A., Chem. Commun.., 1979, 1005.

7. Coruzzi M., Andreeti G.D., Bocchi V., Pochini A., Ungaro R., J. Chem. Soc., Perkin Trans. 2, 1982, 1133.

8. Asfari Z., Bohmer V., Harrowfield J.McB., Calixarenes 2001; Kluwer Academic: Dordrecht, 2001 and references therein.

9. (a) Casnati A., Ungaro R., Calixarenes in spherical metal ion recognition. In Calixarenes in Action; Mandolini L., Ungaro R., Eds; Imperial College: London, 2000, pp 62-84; (b) Casnati A., Della Ca’ N., Sansone F., Ugozzoli F., Ungaro R., Tetrahedron, 2004, 60, 7869; (c) Tu C., Surowiec K., Bartsch A., Tetrahedron, 2007, 63, 4184. 
10. (a) Bohmer V., Ferguson G., Gallagher J.F., Lough A.J., McKervey M.A., Madigan E., Moran M.B., Williams G., J. Chem. Soc., Perkin Trans. 1, 1993, 1521; (b) Oueslati I., Abidi R., Thuery P., Nierlich M., Asfari Z., Harrowfield J., Vicens J., Tetrahedron Letters, 2000, 41, 8263; (c) Kim J.H., Hwang A-R., Chang S-K, Tetrahedron Letters, 2004, 45, 7557.

11. Brewster R.E., Caran K.L., Sasine J.S., Shuker S.B., Current Organic Chemistry, 2004, 8, 867 and references therein.

12. For reviews on double calixarenes, see: (a) Asfari Z., Weiss J., Vicens J., Syntlett., 1993, 719; (b) Ikeda A., Shinkai S., Chem. Rev., 1997, 97, 1713.

13. (a) Yilmaz A., Memon S., Yilmaz M., Tetrahedron, 2002, 58, 7735; (b) Durmaz M., Alpaydin S., Sirit A., Yilmaz M., Tetrahedron: Asymmetry, 2006, 17, 2322; (c) Liang Z., Liu Z., Jiang L., Gao Y., Tetrahedron Letters, 2007, 48, 1629.

14. (a) Liu Y., Zhang N., Zhao B.T., Zhang H.Y., Spectrochimica Acta Part A, 2002, 58, 2889; (b) Singh N., Kumar M., Hundal G., Tetrahedron, 2004, 60, 5393.

15. (a) Alemi A.A., Shaabani B., Acta Chim. Slov., 2000, 47, 363; (b) Liu H., Li B., Liu D., Xu Z., Chemical Physics Letters, 2001, 350, 441.

16. Hwang G.T., Kim B.H., Tetrahedron, 2002, 58, 9019.

17. (a) Arnaud-Neu F., Barrett G., Cremin S., Deasy M., Ferguson G., Harris S.J., Lough A.J., Guerra L., McKervey M.A., Schwing-Weill M.J., Schwinte P., J. Chem. Soc., Perkin Trans. 2, 1992, 1119; (b) Collins E.M., McKervey M.A., J. Chem. Soc., Perkin Trans. 1, 1989, 372.

18. (a) Creaven, B.S., Deasy, M., McKenna, C., Murray, B. A., Tobin, D., Eur. J. Inorg. Chem. 2005, 1282; (b) Creaven, B.S., Deasy, M., Gallagher, J.F., McGinley, J., Murray, B.A., Tetrahedron $2001, \mathbf{5 7}, 8883$.

19. (a) Creaven, B. S., Gernon, T. L., McCormac, T., McGinley, J., Moore, A. M., Toftlund, H., Inorganica Chimica Acta, 2005, 358, 2661; (b) Creaven, B. S., Gernon, T. L., McGinley, J., Moore, A. M., Toftlund, H., Tetrahedron, 2006, 62, 9066; c) Bond, A. D., Creaven, B. S., Donlon, D. F., Gernon, T. L., McGinley, J., Toftlund, H., Eur. J. Inorg. Chem., 2007, 749.

20. Bagatin I.A., de Souza E. S., Ito A.S., Toma H.E., Inorg. Chem. Commun. 2003, 6, 288. 
21. Lhoták P., Shinkai S., Tetrahedron Lett., 1996, 37, 645

22. Bhanthumnavin W., Rojanathanes R., Sukwattanasinitt M., Tuntulani T., Organic Letters 2005, $\underline{6}$, 3401.

23. Liu Y., Wang H., Wang L-H., Li Z., Zhang H-Y., Zhang Q., Tetrahedron, 2003, 59, 7967.

24. Seangpraserthij R., Asfari Z., Arnaud F., Weiss J., Vicens J., J. Inc. Phen. Mol. Rec., 1992, 14, 141.

25. Asfari Z., Arnaud F., Vicens J., J. Org. Chem., 1994, 59, 1741.

26. Liu Y., Zhao B-T., Zhang H-Y., Wada T., Inoue Y., J. Chem. Soc., Perkin Trans. 2, 2001, 1219.

27. Mahajan R.K., Kumar M., Sharma V., Kaur I., Analyst, 2001, 126, 505.

28. Mahajan R.K., Kaur I., Kumar M., Sensors and Actuators B, 2003, 91, 26.

29. Synthesis of 5,11,17,23-tetra-tert-butyl-25,27-bis(propyloxy)benzaldehyde-26,28-dihydroxycalix[4]arene (2): 3-hydroxybenzaldehyde $(0.33 \mathrm{~g}, 2.7 \mathrm{mmol})$ and potassium carbonate $(0.19 \mathrm{~g}$, $1.4 \mathrm{mmol})$ were refluxed for $1 \mathrm{~h}$ in acetonitrile $(30 \mathrm{ml})$, dibromo-calixarene $(\mathbf{1}$, prepared as per Bagatin: reference 10, $1.20 \mathrm{~g}, 1.34 \mathrm{mmol}$ ) was then added and reflux was continued overnight. On cooling the reaction was filtered and the solvent was removed under vacuum. Addition of methanol gave a white precipitate; yield 87.4\% (1.11 g). TLC $\mathrm{R}_{\mathrm{f}} 0.23$ (DCM:hexane 1:1). M.p. 128-130 ${ }^{0} \mathrm{C}$. $\mathrm{C}_{64} \mathrm{H}_{76} \mathrm{O}_{8}$ Found C, 78.78; H, 7.72; requires C 78.98, H 7.87. v max $/ \mathrm{cm}^{-1} 3339(\mathrm{OH}), 1696(\mathrm{CO})$. $\delta_{\mathrm{H}} / \mathrm{ppm} 0.98\left(\mathrm{~s}, 18 \mathrm{H},{ }^{t} \mathrm{Bu}\right), 1.27\left(\mathrm{~s}, 18 \mathrm{H},{ }^{t} \mathrm{Bu}\right), 2.40$ (quin, $\left.4 \mathrm{H}, \mathrm{CH}_{2} \underline{\mathrm{CH}}_{2} \mathrm{CH}_{2}, \mathrm{~J}=6.0 \mathrm{~Hz}\right), 3.30(\mathrm{~d}$, $\left.4 \mathrm{H}, \mathrm{ArC}_{\underline{\mathrm{H}}} \mathrm{H}_{\mathrm{b}} \mathrm{Ar}, \mathrm{J}=13.0 \mathrm{~Hz}\right), 4.14\left(\mathrm{t}, 4 \mathrm{H}, \mathrm{ArOCH}_{2}, \mathrm{~J}=5.9 \mathrm{~Hz}\right), 4.23\left(\mathrm{~d}, 4 \mathrm{H}, \mathrm{ArCH}_{\mathrm{a}} \underline{\mathrm{H}}_{\mathrm{b}} \mathrm{Ar}, \mathrm{J}=\right.$ 13.0 Hz), 4.48 (t, 4H, $\mathrm{CH}_{2} \mathrm{OAr}_{\mathrm{Ald}}, \mathrm{J}=6.2 \mathrm{~Hz}$ ), 6.84 (s, 4H, Calix. ArH), 7.02 (s, 4H, Calix. ArH), 7.08 (s, 2H, 2-ArH), 7.21 (m, 2H, 5-ArH), 7.40 (m, 2H, 4-ArH), 7.45 (bs, 2H, 6-ArH), 7.65 (s, 2H, $\left.\mathrm{OH}), 9.88(\mathrm{~s}, 2 \mathrm{H}, \mathrm{CHO}) . \delta_{\mathrm{C}} / \mathrm{ppm} 29.82\left(\mathrm{CH}_{2} \underline{\mathrm{CH}}_{2} \mathrm{CH}_{2}\right), 30.98\left(\mathrm{C}(\underline{\mathrm{CH}})_{3}\right)_{3}\right), 31.23\left(\mathrm{Ar}_{\underline{C}} \mathrm{H}_{2} \mathrm{Ar}\right), 31.65$ $\left(\mathrm{C}\left(\underline{\mathrm{CH}_{3}}\right)_{3}\right), 33.77\left(\underline{\mathrm{C}}\left(\mathrm{CH}_{3}\right)_{3}\right), 33.95\left(\underline{\mathrm{C}}\left(\mathrm{CH}_{3}\right)_{3}\right), 64.81\left(\mathrm{CH}_{2} \mathrm{CH}_{2} \underline{\mathrm{CH}_{2}}\right), 72.44\left(\underline{\mathrm{CH}_{2}} \mathrm{CH}_{2} \mathrm{CH}_{2}\right), 113.88$

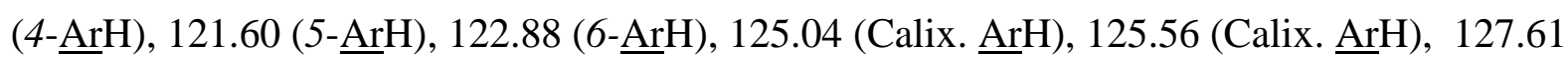
$\left(\underline{\mathrm{ArCH}}{ }_{2} \mathrm{Ar}\right), 129.99$ (2- $\left.\underline{\mathrm{ArH}}\right), 132.64\left(\underline{\mathrm{ArCH}}_{2} \mathrm{Ar}\right), 137.77\left(\underline{\mathrm{Ar}}^{t} \mathrm{Bu}\right), 141.52\left(\underline{\mathrm{Ar}}^{t} \mathrm{Bu}\right), 147.09$ (1-

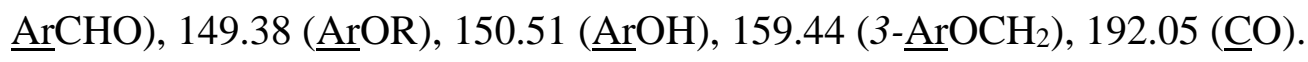


30. Selected data for 3-(pyridin-3-yliminomethyl)phenol (4): yield 78\%; m.p.128-130 ${ }^{0} \mathrm{C} . \mathrm{C}_{12} \mathrm{H}_{10} \mathrm{~N}_{2} \mathrm{O}$ Found C, 72.38; H, 5.10, N, 14.23; requires C, 72.72, H, 5.08; N, 14.13. $v_{\max } / \mathrm{cm}^{-1} 3062(\mathrm{OH})$. $\delta_{\mathrm{H}} / \mathrm{ppm} 7.08(\mathrm{~m}, 1 \mathrm{H}, 2-\mathrm{ArH}), 7.37$ (m, 3H, 4, 5, 6-ArH), 7.63 (m, 1H, 4'-ArH), 7.74 (m, 1H, 2'ArH), 8.49 (m, 2H, CH=N + 5'-ArH), 8.78 (d, 1H, 6'-ArH), 9.39 (bs, 1H, OH). $\delta_{\mathrm{C}} / \mathrm{ppm} 114.10$ (2'-

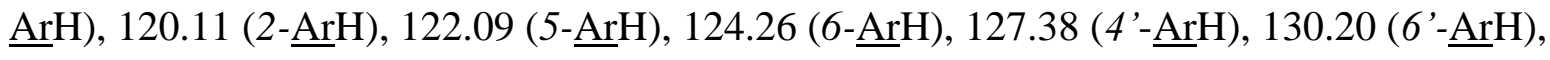
$137.00(\underline{\operatorname{ArCN}}), 143.66$ (6'- $\underline{\text { ArH }}), 146.38$ (5- $\underline{\operatorname{ArH}}), 147.72(\underline{\operatorname{ArN}}), 157.38(\underline{\operatorname{ArOH}}), 161.90(\underline{\mathrm{C}}=\mathrm{N})$. 31. Synthesis of 5,11,17,23-tetra-tert-butyl 25,27-bis[propyloxy(3-pyridin-3-yliminomethyl)phenoxy]26,28-dihydroxycalix[4]arene (3): Schiff base (4) (0.20 g, $1.0 \mathrm{mmol})$ and potassium carbonate $(0.14 \mathrm{~g}, 1.0 \mathrm{mmol})$ were refluxed in acetonitrile $(20 \mathrm{ml})$ for $1 \mathrm{~h}$, dibromo-calixarene $(\mathbf{1})(0.44 \mathrm{~g}$, $0.50 \mathrm{mmol}$ ) was added and reflux was continued for a further $15 \mathrm{~h}$, turning from green to red overnight. The reaction was then cooled, filtered, and solvent evaporated; addition of methanol and cooling gave a red powder; yield 66\% (0.37 g). TLC $\mathrm{R}_{\mathrm{f}} 0.73$ (DCM:hexane 9:1). M.p.120-122 ${ }^{0} \mathrm{C} . \mathrm{C}_{74} \mathrm{H}_{84} \mathrm{~N}_{4} \mathrm{O}_{6}$, found $\mathrm{C}, 79.11 ; \mathrm{H}, 7.30 ; \mathrm{N}, 5.04$; requires $\mathrm{C}, 78.97, \mathrm{H}, 7.52, \mathrm{~N}, 4.98 . v_{\max } / \mathrm{cm}^{-1}$ $3382(\mathrm{OH}), 1627(\mathrm{C}=\mathrm{N}) . \delta_{\mathrm{H}} / \mathrm{ppm} 0.98\left(\mathrm{~s}, 18 \mathrm{H},{ }^{t} \mathrm{Bu}\right), 1.25\left(\mathrm{~s}, 18 \mathrm{H},{ }^{t} \mathrm{Bu}\right), 2.39$ (quin, $4 \mathrm{H}$, $\left.\mathrm{CH}_{2} \underline{\mathrm{CH}}_{2} \mathrm{CH}_{2}, \mathrm{~J}=5.9 \mathrm{~Hz}\right), 3.27\left(\mathrm{~d}, 4 \mathrm{H}, \mathrm{ArC}_{\underline{\mathrm{H}}} \mathrm{H}_{\mathrm{b}} \mathrm{Ar}, \mathrm{J}=12.8 \mathrm{~Hz}\right), 4.12\left(\mathrm{t}, 4 \mathrm{H}, \mathrm{CH}_{2} \mathrm{CH}_{2} \mathrm{CH}_{2}, \mathrm{~J}=5.7\right.$ $\mathrm{Hz}), 4.22\left(\mathrm{~d}, 4 \mathrm{H}, \mathrm{ArCH}_{\mathrm{a}} \underline{\mathrm{H}_{\mathrm{b}}} \mathrm{Ar}, \mathrm{J}=12.8 \mathrm{~Hz}\right), 4.51\left(\mathrm{t}, 4 \mathrm{H}, \mathrm{CH}_{2} \mathrm{CH}_{2} \underline{\mathrm{C}}_{2}, \mathrm{~J}=5.9 \mathrm{~Hz}\right), 6.84(\mathrm{~s}, 4 \mathrm{H}$, Calix. ArH), 7.00 (s, 4H, Calix. ArH), 7.09 (m, 2H, 2-ArH), 7.35 (m, 6H, 4, 5, 6-ArH), 7.57 (m, 2H, 5'-ArH), 7.76 (s, 2H, OH), 8.27 (s, 2H, CH=N), 8.42 (d, 2H, 2'-ArH), 8.46 (d, 2H, 4'-ArH), $\left.8.48\left(\mathrm{~d}, 2 \mathrm{H}, 6^{\prime}-\mathrm{ArH}_{\mathrm{Py}}\right) . \delta_{\mathrm{C}} / \mathrm{ppm} 29.97\left(\mathrm{CH}_{2} \underline{\mathrm{CH}}_{2} \mathrm{CH}_{2}\right), 31.02\left(\mathrm{C}\left(\underline{\mathrm{C}} \mathrm{H}_{3}\right)_{3}\right), 31.59\left(\mathrm{C}^{\left(\underline{\mathrm{C}} \mathrm{H}_{3}\right.}\right)_{3}\right), 31.67$

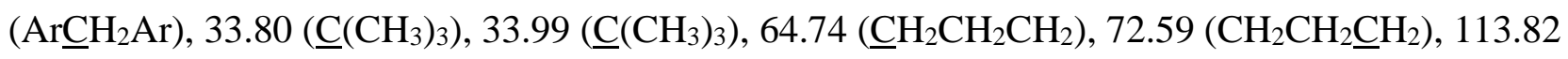

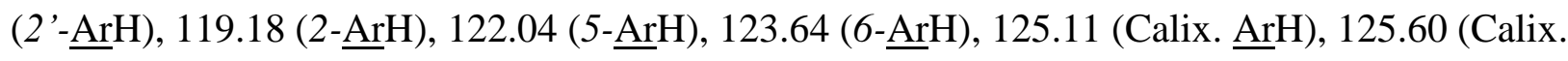
$\underline{\mathrm{ArH}}), 127.59\left(\underline{\mathrm{ArCH}_{2} \mathrm{Ar}}\right), 127.95$ (4' $\left.\underline{-\mathrm{ArH}}\right), 129.83$ (4- $\left.\underline{\mathrm{ArH}}\right), 132.72\left(\underline{\mathrm{ArCH}_{2} \mathrm{Ar}}\right), 137.09(\underline{\mathrm{ArC}}=\mathrm{N})$, $141.53\left(\underline{\mathrm{Ar}}^{t} \mathrm{Bu}\right), 142.58\left(6^{\prime}-\underline{\mathrm{ArH}}\right), 147.00\left(\underline{\mathrm{Ar}}^{t} \mathrm{Bu}\right), 147.13$ (5' $\left.{ }^{\prime} \underline{\mathrm{Ar}} \mathrm{H}\right), 147.75$ (Calix $\left.\underline{\operatorname{ArOR}}\right), 149.39$ $(\underline{\mathrm{ArN}}), 150.60(\underline{\mathrm{ArOH}}), 159.37\left(\underline{\mathrm{ArOCH}_{2}}\right), 161.96(\underline{\mathrm{C}}=\mathrm{N})$.

32. Pitarch M., McKee V., Nieuwenhuyzen M., McKervey M.A., J. Org. Chem., 1998, 63, 234.

33. Matthews S. E, Schmitt P., Felix V., Drew M. G. B., Beer P. D., J. Am. Chem. Soc., 2002, 124, 1341. 
34. a) Kim S K., Wonbo S., Vicens J., Kim J. S., Tetrahedron Lett., 2003, 44, 805; b) Kim S. K., Vicens J., Park K-M., Lee S. S., Kim J. S., Tetrahedron Lett., 2003, 44, 993.

35. Calix-diamine (5) was prepared by reacting the parent calixarene with 2-bromoacetamide, followed by reduction with borane-THF: see Wolf N.J., Georgiev E.M., Yordanov A.T., Whittlesey B.R., Koch H.F., Roundhill D.M., Polyhedron, 1999, 18, 885.

36. Synthesis of Calix-tube (6): calix-diamine (5), (0.33 g, $0.45 \mathrm{mmol})$ and triethylamine (0.070 g, $0.70 \mathrm{mmol})$ were refluxed in methanol (20 ml) for $5 \mathrm{~min}$. Calix-dial (2) $(0.20 \mathrm{~g}, 0.21 \mathrm{mmol})$ was added in methanol (20 ml) over $1 \mathrm{~h}$, slowly yielding a precipitate. After refluxing overnight, then cooling, a white solid was isolated in $89 \%$ yield $(0.31 \mathrm{~g})$. TLC $\mathrm{R}_{\mathrm{f}} 0.47$ (DCM:hexane 1:1). M.p. 222-224 ${ }^{0} \mathrm{C} . \mathrm{C}_{112} \mathrm{H}_{138} \mathrm{~N}_{2} \mathrm{O}_{10}$, found $\mathrm{C}, 80.13 ; \mathrm{H}, 8.11 ; \mathrm{N}, 1.57$; requires $\mathrm{C}, 80.44 ; \mathrm{H}, 8.32, \mathrm{~N}, 1.68$. $\nu_{\max } / \mathrm{cm}^{-1} 3406(\mathrm{OH}), 1603(\mathrm{C}=\mathrm{N}) . \delta_{\mathrm{H}} / \mathrm{ppm} 0.96\left(\mathrm{~s}, 18 \mathrm{H},{ }^{t} \mathrm{Bu}\right), 1.04\left(\mathrm{~s}, 18 \mathrm{H},{ }^{t} \mathrm{Bu}\right), 1.24(\mathrm{~s}, 18 \mathrm{H}$, $\left.{ }^{t} \mathrm{Bu}\right), 1.25\left(\mathrm{~s}, 18 \mathrm{H},{ }^{t} \mathrm{Bu}\right.$ ), 2.39 (quin, $4 \mathrm{H}, \mathrm{CH}_{2} \underline{\mathrm{CH}}_{2} \mathrm{CH}_{2}, \mathrm{~J}=5.9 \mathrm{~Hz}$ ), 3.26 (d, 4H, ArC $\underline{\mathrm{HaH}} \mathrm{H}_{\mathrm{b}} \mathrm{Ar}_{\mathrm{Ald}}, \mathrm{J}=$ $13.0 \mathrm{~Hz}), 3.29\left(\mathrm{~d}, 4 \mathrm{H}, \operatorname{ArC}_{\mathrm{H}_{\mathrm{a}}} \mathrm{H}_{\mathrm{b}} \mathrm{Ar}_{\mathrm{Amine}}, \mathrm{J}=12.8 \mathrm{~Hz}\right), 4.10\left(\mathrm{~m}, 8 \mathrm{H}, \mathrm{ArCH}_{\mathrm{a}} \underline{\mathrm{H}}_{\mathrm{b}} \mathrm{Ar}_{\mathrm{Amine}}+\right.$ $\mathrm{C}_{2} \mathrm{CH}_{2} \mathrm{CH}_{2}$ ), 4.28 (m, 16H, $\mathrm{ArCH}_{2} \underline{\mathrm{H}}_{\mathrm{b}} \mathrm{Ar}_{\mathrm{Ald}}+\mathrm{CH}_{2} \mathrm{CH}_{2} \underline{\mathrm{C}}_{2}+\mathrm{NC}_{2} \underline{\mathrm{C}}_{2} \mathrm{O}$ ), 6.81 (s, 4H, Calix. $\left.\mathrm{ArH}_{\mathrm{Ald}}\right), 6.89$ (s, 4H, Calix. ArH $\mathrm{Amine}_{\text {) }}, 6.93$ (m, 2H, 2-ArH), 6.95 (s, 4H, Calix. ArH Ald $), 6.96$ (s, 4H, Calix. ArH $\mathrm{Amine}_{\text {) }} 7.21(\mathrm{t}, 2 \mathrm{H}, 5-\mathrm{ArH}), 7.31\left(\mathrm{~s}, 4 \mathrm{H}, \mathrm{OH}_{\mathrm{Amine}}+6-\mathrm{ArH}\right), 7.44(\mathrm{~m}, 2 \mathrm{H}, 4-\mathrm{ArH})$,

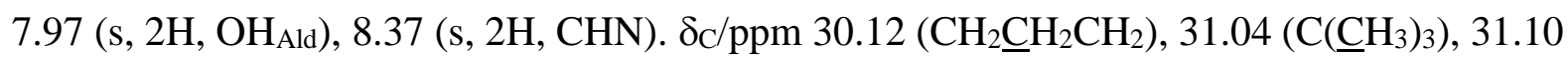
$\left(\mathrm{C}\left(\underline{\mathrm{CH}}_{3}\right)_{3}\right), 31.69\left(\mathrm{C}\left(\underline{\mathrm{CH}}_{3}\right)_{3}\right), 31.71\left(\mathrm{C}\left(\underline{\mathrm{CH}}_{3}\right)_{3}\right), 31.97\left(4 \mathrm{Ar} \underline{\mathrm{C}} \mathrm{H}_{2} \mathrm{Ar}\right), 33.79\left(2 \underline{\mathrm{C}}\left(\mathrm{CH}_{3}\right)_{3}\right), 33.92$ $\left(\underline{\mathrm{C}}\left(\mathrm{CH}_{3}\right)_{3}\right), 34.04\left(\underline{\mathrm{C}}\left(\mathrm{CH}_{3}\right)_{3}\right), 60.47\left(\mathrm{NC}_{2} \mathrm{CH}_{2} \mathrm{O}\right), 64.53\left(\mathrm{OCH}_{2} \mathrm{CH}_{2} \mathrm{CH}_{2} \mathrm{O}\right), 73.04$ $\left(\mathrm{OCH}_{2} \mathrm{CH}_{2} \underline{\mathrm{CH}}_{2} \mathrm{O}\right), 75.48\left(\mathrm{NCH}_{2} \underline{\mathrm{CH}}_{2} \mathrm{O}\right), 113.47$ (6- $\left.\underline{\mathrm{ArH}}\right), 117.80$ (5- $\left.\underline{\mathrm{ArH}}\right), 120.94$ (4- $\left.\underline{\mathrm{ArH}}\right), 124.93$ (Calix. $\left.\underline{A r H}_{\text {Amine }}\right), 125.10$ (Calix. $\left.\underline{\text { ArH }}{ }_{\text {Ald }}\right), 125.46$ (Calix. $\left.\underline{\operatorname{ArH}}{ }_{\text {Ald }}\right), 125.61$ (Calix. $\left.\underline{A r H}_{\text {Amine }}\right), 127.62$

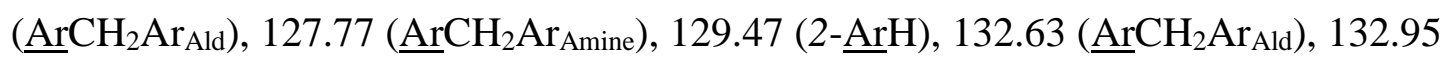

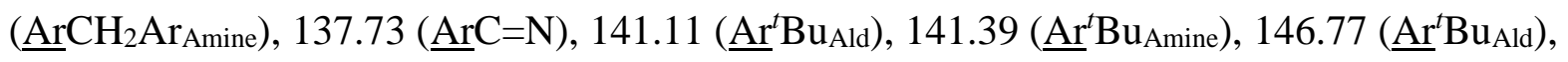

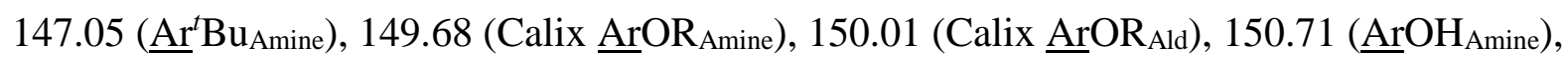
$150.73\left(\underline{\mathrm{ArOH}}_{\mathrm{Ald}}\right), 159.07\left(\underline{\mathrm{ArOCH}_{2}}\right), 163.86(\underline{\mathrm{C}}=\mathrm{N})$. 


\section{Captions}

Scheme 1

Synthesis of calix-dial (2) and calix-Schiff (3)
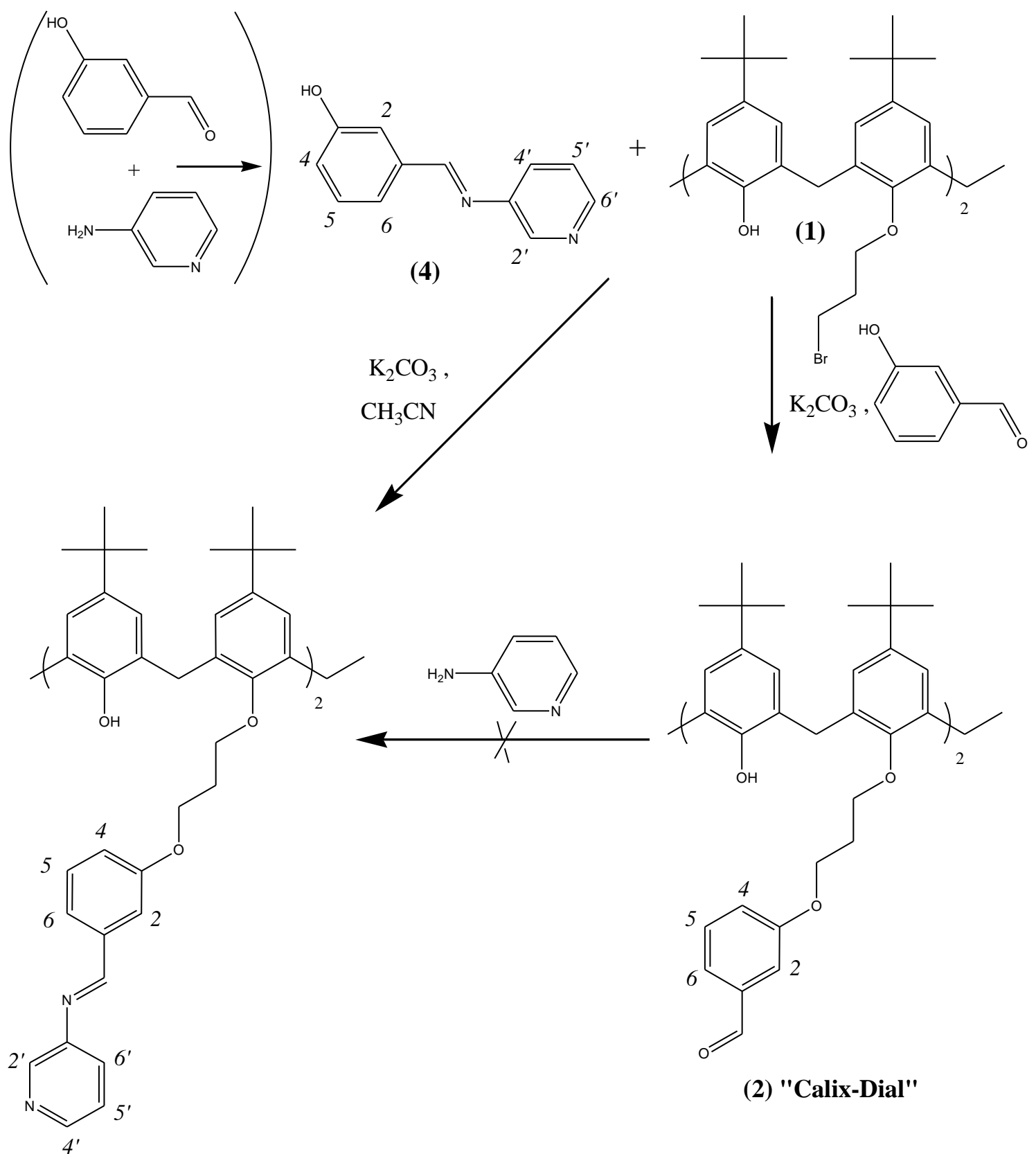

(3)"Calix-Schiff"

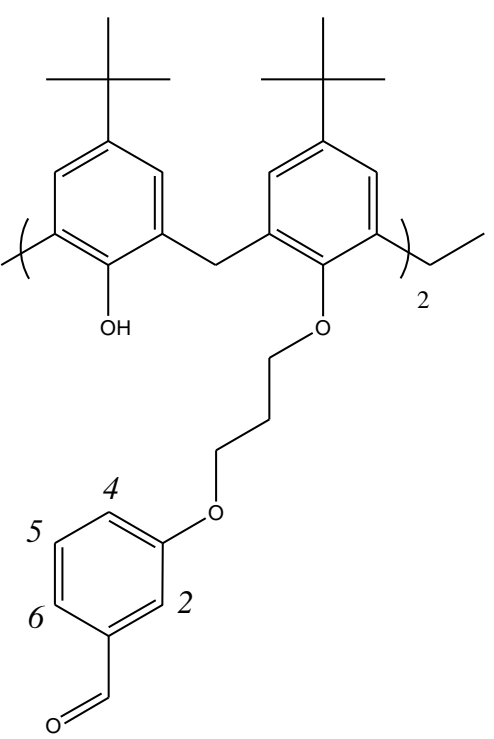

(2) "Calix-Dial" 
Scheme 2

Synthesis calix-tube (6)<smiles>CCCCOc1c(CC)cc(C(C)(C)C)cc1Cc1cc(C(C)(C)C)cc(C(C)(C)C)c1O</smiles>

(5) "Calix-Diamine"

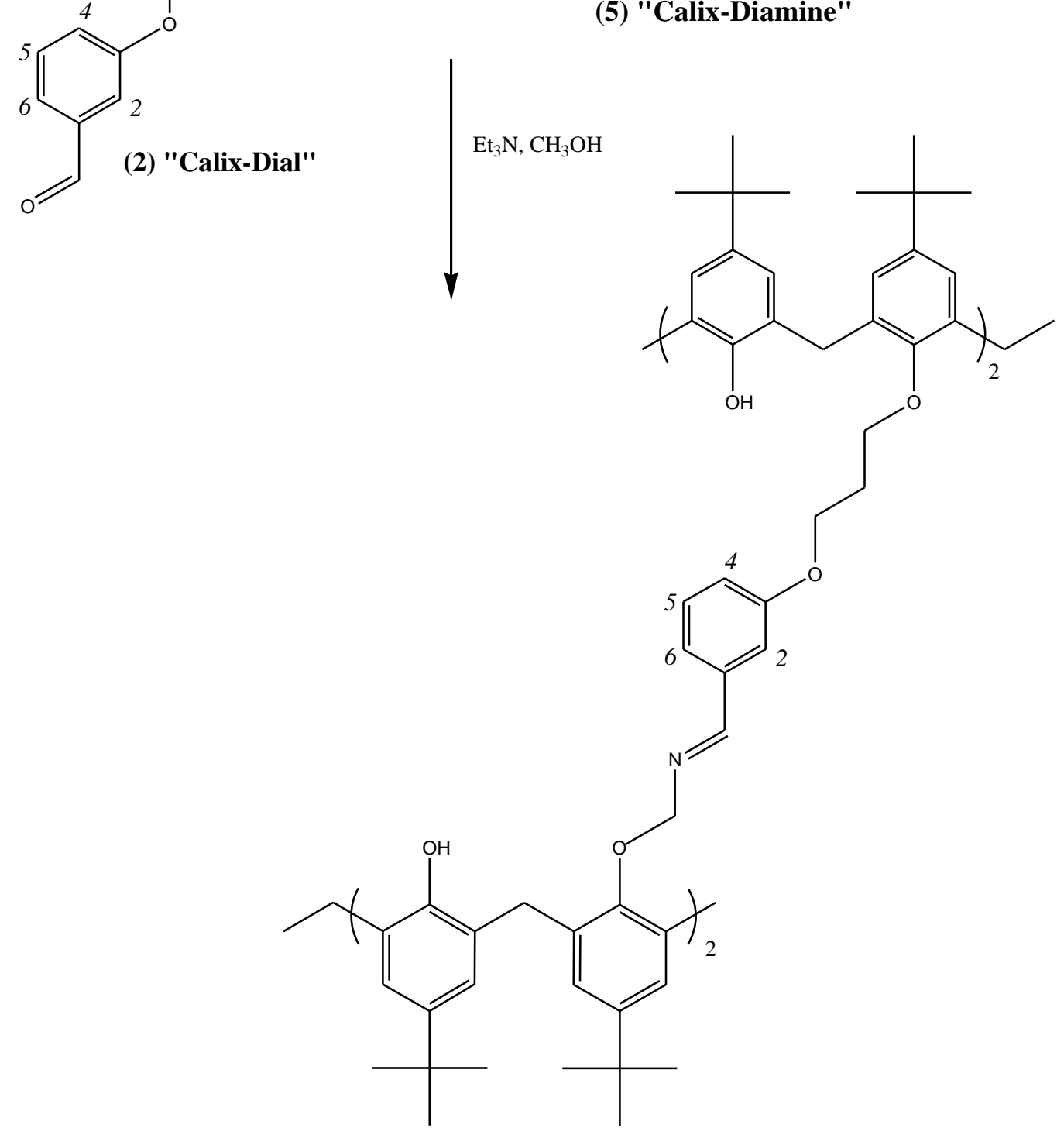

(6) "Calix-Tube" 
Figure 1: ${ }^{1} \mathrm{H}$ NMR Stack showing uncomplexed (top) and Complexed (bottom) (3), aromatic region (both in $\mathrm{CDCl}_{3}$ solution).

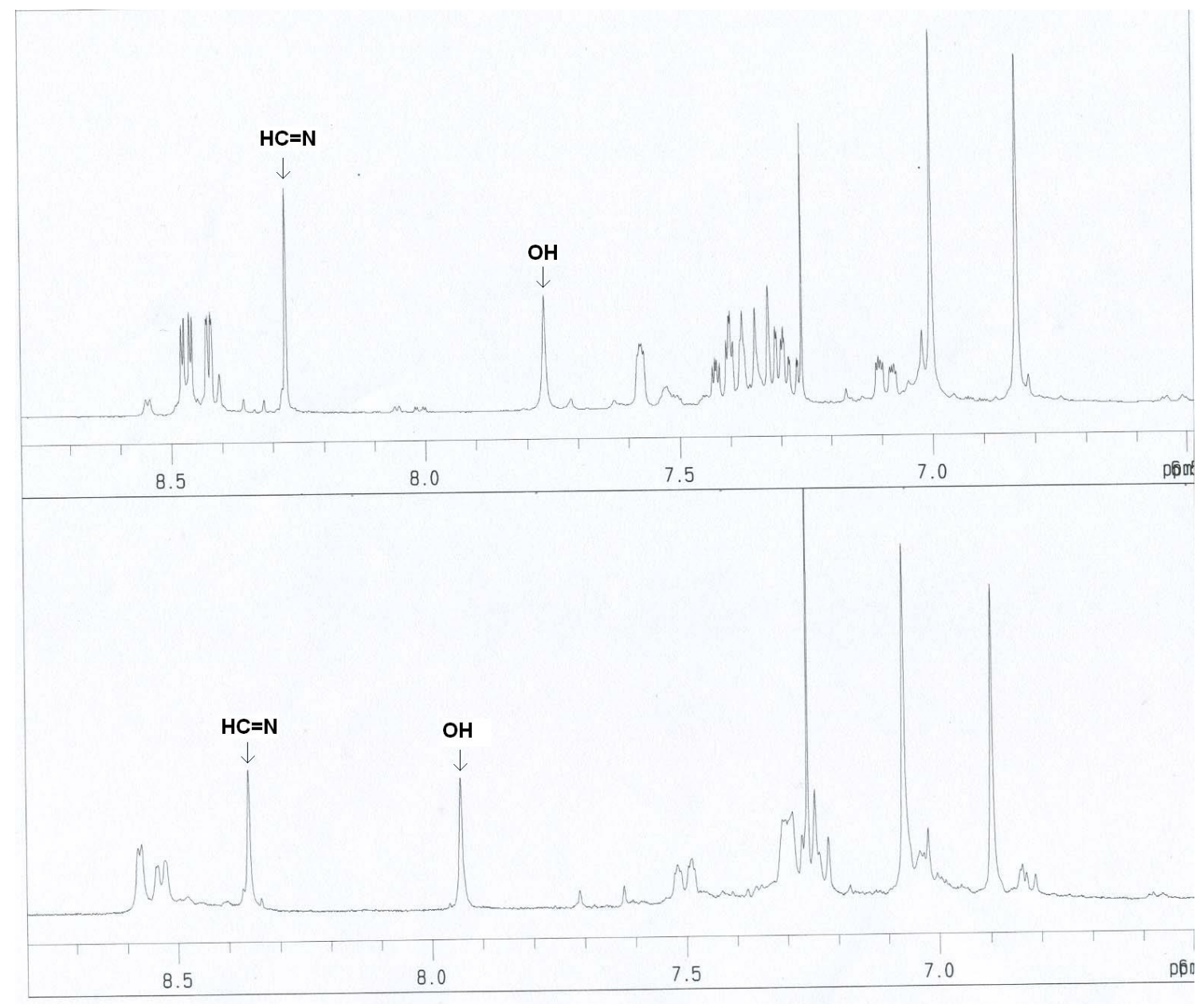


Figure 2: ${ }^{1} \mathrm{H}$ NMR Stack showing uncomplexed (top) and Complexed (bottom) (6); aromatic region (both in $\mathrm{CDCl}_{3}$ solution).

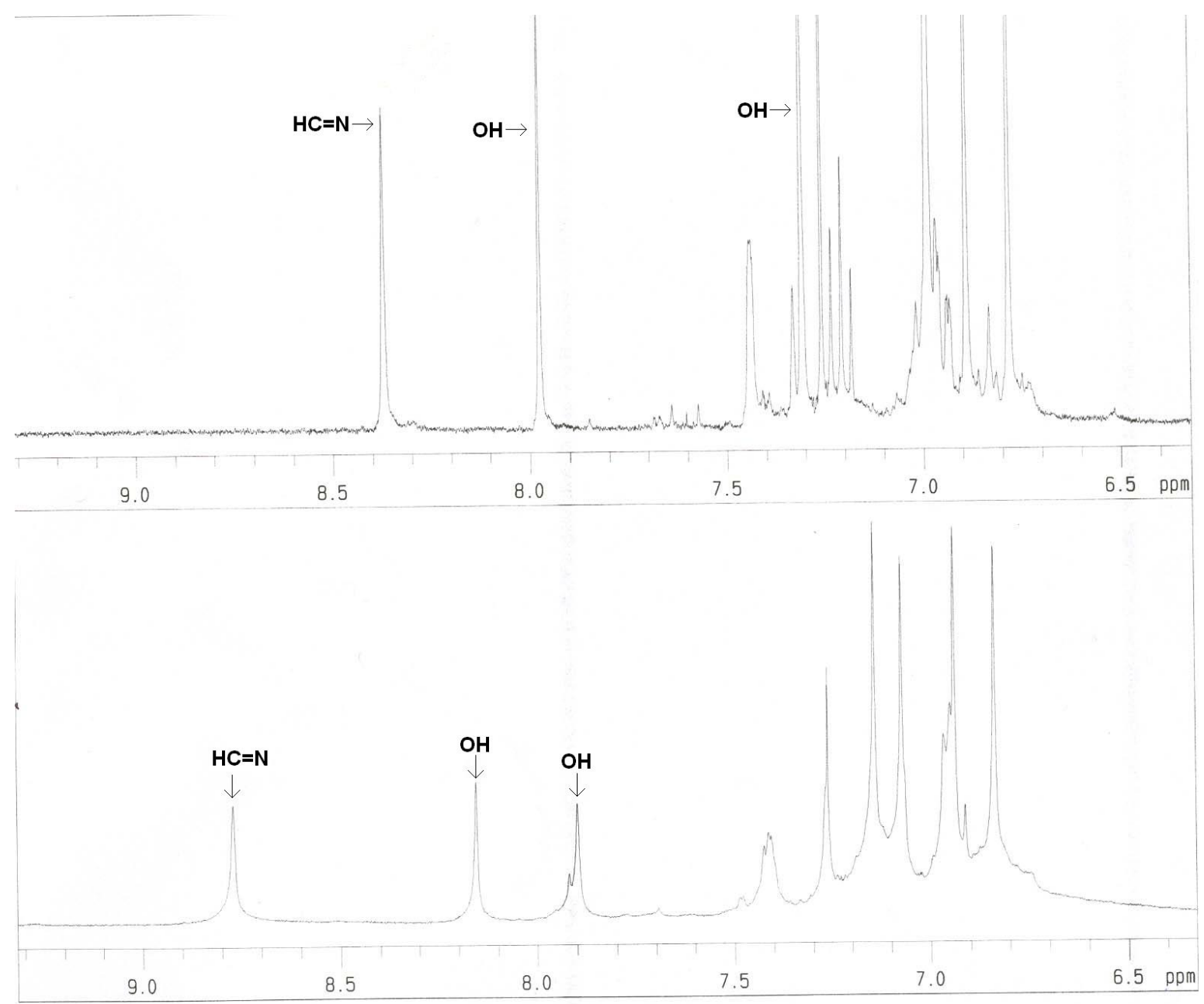

